Aporte Santiaguino 11 (1), enero-junio 2018: 119-130

Online ISSN 2616-9541X

Print ISSN: 2070-836X

\title{
Sistema de control gubernamental y la corrupción política, económica y social en el Gobierno Regional de Áncash, 2011-2014
}

System of governmental control and political, economic and social corruption in the Ancash Regional Government, 2011-2014

\author{
Javier Hidalgo Mejía ${ }^{1}$, Alcides Medina Ortega ${ }^{1}$, José Castro Palma ${ }^{1}$ y \\ Richard Pasco Ames ${ }^{1}$
}

\section{RESUMEN}

El propósito de la investigación fue evaluar la incidencia del sistema de control gubernamental en la lucha contra la corrupción política, económica y social del Gobierno Regional de Áncash, 2011 - 2014. Para lo cual se planteó el problema: ¿Cómo incide el sistema de control gubernamental en la lucha contra la corrupción política, económica y social?, cuya hipótesis es: el control previo, simultáneo y posterior incide de manera insuficiente en la reducción de la corrupción política, económica y social. Investigación aplicada con diseño no experimental, población y muestra 450 y 152 servidores, técnicas e instrumentos aplicados, encuesta, análisis documental y entrevista, cuestionario, ficha de análisis documental y guía de entrevista. Los resultados corroboran que el sistema de control gubernamental incide de manera insuficiente en la corrupción del Gobierno Regional de Áncash. Se concluye que el control previo incide insuficientemente en la reducción de la corrupción política, por ende, el control previo y la corrupción política se relacionan; el control simultáneo impacta exiguamente en la disminución de la corrupción económica. En consecuencia, el control simultáneo y la corrupción económica se corresponden mutuamente; y el control posterior incide escasamente en la contracción de la corrupción social.

Palabras clave: control previo; simultáneo y posterior; corrupción política; económica y social.

\footnotetext{
1 Universidad Nacional Santiago Antúnez de Mayolo. Huaraz, Perú.

(C) Los autores. Este artículo es publicado por la Revista Aporte Santiaguino de la Universidad Nacional Santiago Antúnez de Mayolo. Este es un artículo de acceso abierto, distribuido bajo los términos de la Licencia Creative Commons Atribución-NoComercial-CompartirIgual 4.0 Internacional. (http://creativecommons.org/licenses/ by-nc-sa/4.0/), que permite el uso no comercial, distribución y reproducción en cualquier medio, siempre que la obra original sea debidamente citada.
} 


\section{ABSTRACT}

The purpose of this research was to evaluate the influence of the government control system in the fight against political, economic and social corruption of the Regional Government of Ancash, 2011 - 2014. For which the problem statement was: How does the system of governmental control impact on the fight against political, economic and social corruption? The hypothesis statement was prior, simultaneous and the subsequent control may have an insufficient impact on the reduction of political, economic and social corruption. The type of research was applied, with nonexperimental design, with a population and sample of 450 and 152 servers respectively, techniques and applied instruments, survey, documentary analysis and interview, questionnaire, document analysis sheet and interview guide. The results confirm that the government control system has an insufficient impact on the corruption of the Regional Government of Ancash. The conclusion is that prior control has an insufficient impact on the reduction of political corruption, therefore prior control and political corruption are related; simultaneous control has a negligible impact on the reduction of economic corruption. Consequently, simultaneous control and economic corruption correspond to each other; and the subsequent control scarcely affects the contraction of social corruption.

Keywords: prior control; simultaneous and subsequent; political corruption; economic and social.

\section{INTRODUCCIÓN}

En Áncash las denuncias por corrupción de funcionarios e instituciones públicas superan las 1,200 y el 60\% involucra al Gobierno Regional. Desde el 2010 al 2014, tuvo un ingreso de S / 5,416’000,000, y solo el Gobierno Regional manejó en los últimos cinco años S/ 1,422’000,000, según cifras del Ministerio de Economía y Finanzas. A pesar de la bonanza, hoy Áncash tiene a su expresidente regional César Álvarez tras las rejas, a un vicepresidente regional con arresto domiciliario, a funcionarios regionales en la cárcel y otros prófugos, todos por denuncias de corrupción.

Khoury (2014) manifestó que más de 500 acciones de control se realizaron en la gestión de César Álvarez. Sin embargo, la corrupción y la ola de violencia han convertido a la región Áncash en un problema generalizado, habiendo la Contraloría reportado que, de los 2,752 funcionarios públicos denunciados durante su gestión, 300 recibieron una sentencia. De los cuales apenas 11 obtuvieron pena efectiva de cárcel; mientras que el resto, condenas suspendidas. 
El Congreso (2002) explica que el control gubernamental es:

La supervisión, vigilancia y verificación de los actos y resultados de la gestión pública, en atención al grado de eficiencia, eficacia, transparencia y economía en el uso y destino de los recursos y bienes del Estado, así como del cumplimiento de las normas legales y de los lineamientos de política y planes de acción, evaluando los sistemas de administración, gerencia y control, con fines de su mejoramiento a través de la adopción de acciones preventivas y correctivas pertinentes.

Entonces el Gobierno Regional de Áncash debió ser fiscalizado de manera permanente e integral, a fin de promover una cultura de uso racional de los recursos públicos. Contrariamente, en el Gobierno Regional de Áncash, ni el control interno, ni el control externo son lo suficientemente efectivos, máxime si se sabe que la capacidad operativa de Contraloría General de la República y del Órgano Regional de Control Institucional, resulta extremadamente limitado, por lo que la ejecución de servicios de control no son los suficientes.

En ese contexto, la corrupción política, económica y social se ha instaurado en la entidad, debido a que los controles previo, simultáneo y posterior no son lo suficientemente efectivos, por lo que su accionar se sujeta a la ejecución de un número reducido de labores de control, lo que no le permite examinar mayores procesos, actividades y tareas; con el añadido de que los pocos informes especiales caen a nivel de Fiscalía y del Poder Judicial; situación que es concordarte con lo descrito por el Instituto de Defensa Legal que sostiene que antes de la detención del ex presidente regional de Ancash, ninguna denuncia penal contra él, o sus cómplices, había prosperado en el Ministerio Público. Ante esa realidad, se tiene como problema de investigación a lo siguiente: ¿cómo incide el sistema de control gubernamental, en la lucha contra la corrupción política, económica y social del Gobierno Regional de Áncash, 2011-2014?; ¿Cómo incidió el control previo, en la lucha contra la corrupción política?, ¿de qué manera incidió el control simultáneo en la lucha contra la corrupción económica? y ¿cómo incidió el control posterior, en la lucha contra la corrupción social?

Los objetivos se orientan a demostrar cómo incidió el sistema de control gubernamental en la lucha contra la corrupción política, económica y social del Gobierno Regional de Áncash, 2011-2014; analizar cómo incidió el control previo en la lucha contra la corrupción política del Gobierno Regional de Áncash; investigar de qué manera incidió el control simultáneo en la lucha contra la corrupción económica del Gobierno Regional de Áncash y analizar cómo incidió el control posterior en la lucha contra la corrupción social del Gobierno Regional de Áncash.

Los antecedentes sobre la corrupción desde una perspectiva económica, establecen que las estrategias legales que existen se basan en la idea, noción que el comportamiento corrupto puede verse limitado por la estricta aplicación de la ley; así mismo, se tiene que para combatir la corrupción no es necesario dictar nuevas leyes sino más bien 
cambiar las políticas existentes, enmarcados en el tema de control; la tesis del "control gubernamental y el sistema de control interno en el Perú: análisis crítico", señala que el control gubernamental es tarea de todos, se ejecuta bajo dos (2) modalidades o momentos; la primera se denomina "control interno" y es responsabilidad de todos los miembros de una organización pública, la segunda modalidad se denomina "control externo" que es ejecutado tanto por la Contraloría General, por los auditores designados por esta; de igual manera, se advierte que el control previo previene los problemas de manera anticipada, tiene lugar antes de que se desarrolle la actividad, el control concurrente tiene lugar mientras se desarrolla la actividad y el control posterior, tiene lugar después de la acción; la corrupción política es el mal uso o el abuso del poder público para beneficio personal y privado, por lo general se apunta a los gobernantes o los funcionarios elegidos o nombrados que se dedican a aprovechar los recursos del Estado para enriquecerse o beneficiar a parientes o amigos; con relación a la corrupción económica, se tiene que la economía de la ley es entonces fundamental para entender el problema de la corrupción. Esta es una consecuencia de una mala economía de la ley; respecto a la corrupción social dice que tiene una segunda hermana olvidada ya que, para que exista, no necesita solo de corruptibles sino también de corruptores.

\section{MATERIALES Y MÉTODOS}

El tipo de estudio fue el aplicado; diseño de investigación, no experimental; y nivel, correlacional. La población estuvo comprendida por el número de autoridades, directivos, funcionarios y servidores, que en promedio suma 450. El tamaño de la muestra se definió en 152 servidores públicos. Las técnicas e instrumentos de recolección de datos empleados fueron la encuesta, análisis documental, guía de entrevistas, cuestionario, fichas resumen fichas de entrevista; las técnicas de procesamiento y análisis de datos empleados fueron el SPSS y el excel.

\section{RESULTADOS}

\section{Análisis de la incidencia del control previo en la lucha contra la corrupción política}

El 9\% de los encuestados que manifestaron que no se cumple con la autorización previa de Contraloría para la ejecución y pago de presupuestos adicionales de obra y mayores prestaciones de supervisión, son los que consideran que se cumple de forma aceptable y en mayor grado que los funcionarios o servidores públicos que, abusando de su cargo, obligan o inducen a una persona a dar o prometer indebidamente, para sí o para otro, un bien o un beneficio patrimonial. El 14\% de los investigados que manifestaron que no se cumple con la opinión previa de Contraloría sobre garantías 
otorgadas al Gobierno Regional de Áncash, son los que consideran que se cumple en mayor grado que los servidores públicos incurren en acción y/u omisión en el ejercicio de sus funciones con el fin de obtener ilícitamente beneficios para sí mismo o para un tercero. El 16\% que sostuvieron que no se cumple la función de Contraloría de emitir opiniones vinculantes sobre contrataciones de orden interno exonerado de procesos de selección, son los que consideran que se cumple en mayor grado la impunidad de los actos de corrupción que involucran a servidores del Gobierno Regional de Áncash.

\section{Investigación de la incidencia del control simultáneo en la lucha contra la co- rrupción económica}

El 13\% manifestó que no se cumple la obligación que el sistema de control tiene para realizar acciones simultáneas evaluando una o más actividades en ejecución de un proceso en curso, son los que afirman que se cumple plenamente que el servidor público que, abusando de su cargo, incrementa ilícitamente su patrimonio respecto de sus ingresos legítimos. El 16\% que respondió que se cumple de forma insuficiente que el sistema de control realice la orientación de oficio, alertando al titular de la entidad sobre situaciones que puedan generar riegos, son los que señalan que se cumple en mayor grado que el servidor público se apropia o usa, para sí o para otro, caudales cuya percepción, administración o custodia le estén confiadas por razón de su cargo. El 19\% que reveló que se cumple de forma insuficiente que el sistema de control realiza visitas de control, presenciando la entrega de bienes, prestación de servicios o ejecución de obras, son los que señalan que se cumple en mayor grado el incremento de la capacidad de gasto de los funcionarios o servidores públicos.

\section{Análisis de la incidencia del control posterior en la lucha contra la corrupción social}

El 15\% que confesó que se cumple de forma aceptable que el sistema de control realiza auditorías a los estados financieros y a la información presupuestaria, son los que precisan que se cumple en mayor grado que la corrupción es producida por una alteración patológica de los valores colectivos de los funcionarios. Del 23\% de los que sostuvieron que no se cumple que el sistema de control realiza auditorías de desempeño para determinar la eficacia, eficiencia, economía y calidad de la producción y entrega de bienes y servicios, son los que precisan que se cumple en mayor grado que la ineficiencia del estado se origina por los trámites y procedimientos burocráticos que reducen la agilidad y transparencia de la gestión pública. El 22\% que manifestó que no se cumple que el sistema de control realiza auditorías de cumplimiento para determinar si la entidad aplica la normativa, disposiciones internas y estipulaciones contractuales, son los que señalan que se cumple en mayor grado que es escasa la participación ciudadana en la vigilancia de los actos de los servidores públicos. 


\section{Resultados del análisis documental}

\section{Análisis de la relación del sistema de control gubernamental y la corrupción}

Durante el periodo 2011 - 2014, el sistema nacional de control no llevó a cabo los servicios de control previo tales como la «autorización previa de contraloría para la ejecución y pago de presupuestos adicionales de obra y mayores prestaciones de supervisión»; «la contraloría informa previamente sobre las operaciones, fianzas, avales y otras garantías, incluyendo proyectos de contrato, que en cualquier forma comprometan su crédito o capacidad financiera, sea que se trate de negociaciones en el país o en el exterion»; y «la contraloría emite opinión vinculante sobre contrataciones de bienes, servicios u obras que tengan el carácter de orden interno exoneradas de licitación pública, concurso público o adjudicación directa». En ese contexto, la Contraloría General de la República, no realizó acciones simultáneas, orientaciones de oficio, ni visitas de control; sin embargo, el Órgano Regional de Control Institucional del Gobierno Regional de Ancash ejecutó 60 acciones simultáneas, 42 orientaciones de oficio, y 38 visitas de control. Por otro lado, las sociedades de auditoría ejecutaron 4 auditorías a la información financiera y a la información presupuestaria; la Contraloría General de la República y el Órgano Regional de Control Institucional no realizaron ninguna auditoría de desempeño; la Contraloría General de la República realizó 14 auditorías de cumplimiento y el Órgano Regional de Control Institucional 42, de las cuales, 19 corresponden a informes especiales por responsabilidad civil y penal y en 23 se determinaron responsabilidades administrativas. Los 200 servicios de control ejecutados por el sistema de control gubernamental se aplicaron a solo una parte de los siguientes procesos de adquisición de bienes, servicios, obras y consultorías de obras:

Tabla 1. Adquisiciones realizadas por el Gobierno Regional de Áncash

\begin{tabular}{|c|c|c|c|c|c|c|c|c|c|c|c|}
\hline \multirow[t]{2}{*}{$\mathrm{N}^{\circ}$} & \multirow{2}{*}{$\begin{array}{l}\text { Descrip- } \\
\text { ción }\end{array}$} & \multicolumn{7}{|c|}{ Cantidad - proceso de selección } & \multicolumn{3}{|l|}{ Montos (s/) } \\
\hline & & 2011 & 2012 & 2013 & 2014 & Total & 2011 & 2012 & 2013 & 2014 & Total \\
\hline 1 & Bienes & 107 & 111 & 76 & 15 & 309 & $31,001,031$ & $13,313,951$ & $21,899,089$ & $1,301,455$ & $67,515,525$ \\
\hline 2 & Servicios & 67 & 171 & 48 & 10 & 298 & $41,161,617$ & $28,284,882$ & $11,159,564$ & $1,359,702$ & $81,965,765$ \\
\hline 3 & Obras & 71 & 62 & 11 & 27 & 171 & $262,760,978$ & $422,827,963$ & $30,699,763$ & $115,639,275$ & $831,927,979$ \\
\hline 4 & $\begin{array}{c}\text { Consul- } \\
\text { toria de } \\
\text { obras }\end{array}$ & 86 & 121 & 50 & 21 & 278 & $10,071,621$ & $19,866,949$ & $21,348,893$ & $3,513,059$ & $54,800,522$ \\
\hline & Total & 331 & 467 & 185 & 73 & 1,056 & $344,995,246$ & $484,293,746$ & $85,107,309$ & $121,813,490$ & $1,036,209,790$ \\
\hline
\end{tabular}

Fuente: Portal del Organismo Supervisor del Estado 
Así mismo, las recomendaciones derivadas de los informes de auditoría realizadas por la Contraloría General de la República, Órgano Regional de Control Institucional y Sociedades de Auditoría, tienen la siguiente composición:

Tabla 2. Estado situacional de las recomendaciones derivadas de las auditorias de cumplimiento - examenes especiales

\begin{tabular}{|c|c|c|c|c|c|c|}
\hline \multirow[t]{2}{*}{$\mathrm{N}^{\circ}$} & \multirow[t]{2}{*}{ Servicios de control } & \multicolumn{5}{|c|}{ Períodos } \\
\hline & & 2011 & 2012 & 2013 & 2014 & Total \\
\hline 1 & Pendiente & 221 & 265 & 165 & 312 & 963 \\
\hline 2 & En proceso & 504 & 481 & 634 & 541 & 2,160 \\
\hline 4 & Retomada & 34 & 34 & 34 & 34 & 136 \\
\hline 5 & No aplicable & 8 & 8 & 8 & 8 & 32 \\
\hline & Total & 2,778 & 2,800 & 2,854 & 2,909 & 3,291 \\
\hline
\end{tabular}

Fuente: Gobierno Regional de Áncash

El Gobierno Regional de Áncash, al 31 de diciembre de 2014, tenía 2,160 recomendaciones en situación de "en proceso" y 963 recomendaciones en condición de "pendiente", hecho que no incide en la reducción de la corrupción ni mejora de la gestión, por el alto grado de omisión en la implementación de recomendaciones.

\section{Resultados de la entrevista a servidores y usuarios del Gobierno Regional de Áncash}

El 60\% de los entrevistados manifestaron que las labores de control realizadas por el Sistema de Control Gubernamental fueron insuficientes, el 22\% precisa que fue regular y el 18\% que fue suficiente. El 32\% precisó que la causa que limita la implementación de las recomendaciones se debe a la negligencia de los funcionarios, el $26 \%$ manifestó que se debió al cambio permanente de funcionarios, el 21\% cree que fue por premeditación y el 16\% expresó que se debe a que no se sanciona a los funcionarios que incumplen la implementación de las recomendaciones derivadas de los informes de control; y el 25\% de los entrevistados sostiene que la causa de la corrupción en la entidad estudiada, fue la impunidad, el $20 \%$ explica que se debió a la falta de valores, el 17\% expresó que fue el uso indebido de caudales, el 11\% sostiene que se generó por la acción u omisión de los funcionarios y/o servidores y el 10\% cree se debió al incremento ilícito del patrimonio de los servidores. 


\section{DISCUSIÓN}

Robbins y Coulter (1994) plantean que el «control previo o preventivo previene los problemas de manera anticipada, tiene lugar antes que se desarrolle la actividad. La clave de los controles previos es tomar la acción administrativa antes de que surja un problema». Así mismo Sayed y Bruce (1998) aseveran que «la corrupción es el mal uso o el abuso del poder público para beneficio personal y privado, entendiendo que este fenómeno no se limita a los funcionarios públicos»; sin embargo, de los resultados se tiene que el $9 \%$ de los encuestados que manifestaron que no se cumple con la autorización previa de Contraloría para la ejecución y pago de presupuestos adicionales de obra y mayores prestaciones de supervisión, son los que consideran que se cumple de forma aceptable y en mayor grado que los funcionarios o servidores públicos que, abusando de su cargo, obligan o inducen a una persona a dar o prometer indebidamente, para sí o para otro, un bien o un beneficio patrimonial; el 14\% que manifestó que no se cumple con la opinión previa de Contraloría sobre garantías otorgadas, son los que consideran que se cumple en mayor grado que los servidores públicos incurren en acción y/u omisión en el ejercicio de sus funciones con el fin de obtener ilícitamente beneficios para sí mismo o para un tercero; y el 16\% que manifestó que no se cumple la función de Contraloría de emitir opinión vinculante sobre contrataciones de orden interno exoneradas de procesos de selección, son los que consideran que se cumple en mayor grado la impunidad de los actos de corrupción que involucran a servidores. El análisis documental reveló que la Contraloría General de la República no otorgó la autorización previa a la ejecución y pago de los presupuestos adicionales de obra pública y de las mayores prestaciones de supervisión en los casos cuyos montos excedan a los previstos; no informó previamente sobre las operaciones, fianzas, avales y otras garantías que otorgó el Gobierno Regional de Áncash; y no emitió opinión vinculante sobre contrataciones de bienes, servicios u obras que tengan el carácter de orden interno exoneradas de procesos de selección. El 60\% manifestó que las labores de control realizadas por el Sistema de Control son insuficientes, el 22\% precisó que es regular y el 18\% que es suficiente; lo que es concordante con los resultados de la investigación efectuada por Cárdenas (2010), quien concluye que «la Contraloría General de la República sólo puede cumplir eficazmente sus funciones si son independientes y se encuentren protegidas contra influencias exteriores».

El control concurrente tiene lugar mientras se desarrolla la actividad. En este caso, la administración puede corregir los problemas antes que se vuelvan muy costosos o peligrosos. La forma más conocida de control concurrente es la supervisión directa, el administrador puede vigilar de manera concurrente las acciones de sus subordinados y corregir los problemas a medida que se presentan (Robbins y Coulter, 1994). 
Así mismo, se argumenta que «la economía de la ley es entonces fundamental para entender el problema de la corrupción. Esta es una consecuencia de una mala economía de la ley. Cuando el costo de la legalidad excede a su beneficio, la ley se incumple» (North, 1995). Sin embargo, se tiene que los resultados de la investigación, dado a que el 13\% de los encuestados que manifestaron que no se cumple la obligación que el sistema de control realiza acciones simultáneas evaluando una o más actividades en ejecución de un proceso en curso, son los que sostienen que se cumple plenamente que el servidor público que, abusando de su cargo, incrementa ilícitamente su patrimonio respecto de sus ingresos legítimos; el 16\% que respondió que se cumple de forma insuficiente que el sistema de control realiza orientaciones de oficio, alertando al titular de la entidad sobre situaciones que puedan generar riesgos, son los que señalan que se cumple en mayor grado que el servidor público se apropia o usa, para sí o para otro, caudales cuya percepción, administración o custodia le estén confiados por razón de su cargo; y el 19\% respondió que se cumple de forma insuficiente que el sistema de control realiza visitas de control, presenciando la entrega de bienes, prestación de servicios o ejecución de obras, son los que señalan que se cumple en mayor grado el incremento indebido de la capacidad de gasto de los funcionarios o servidores públicos. Del análisis documental se infiere que la Contraloría no realizó servicios de control simultáneo; sin embargo, el ORCI ejecutó 140 servicios de control simultáneo, siendo insuficiente los servicios de control para la cantidad de operaciones ejecutadas por el Gobierno Regional de Áncash. De las entrevistas se tiene que el 32\% señaló que la causa que limitó la implementación de las recomendaciones fue la negligencia de los funcionarios, el 26\% manifestó que se debió al cambio permanente de funcionarios, el $21 \%$ cree que fue por premeditación y el 16\% expresó que se debió a que no se sanciona a los funcionarios que incumplen la implementación de las recomendaciones, lo que implica que, además de lo reducido que resulta la cantidad de servicios de control, sus recomendaciones no son implementadas en su totalidad, lo que significa que el control no solo es insuficiente, sino también, no es útil en la medida que no se implementan las recomendaciones de auditoría.

El «control posterior se basa en la retroalimentación, tiene lugar después de la acción, su desventaja es que para cuando el administrador tiene la información, el daño ya está hecho. Sin embargo, en muchas actividades, el control posterior es el único tipo de control disponible» (Robbins y Coulter 1994). Con relación a la corrupción social, se tiene que es «una pandemia gubernamental latinoamericana, cáncer de nuestras democracias, tiene una segunda hermana olvidada, y es que para que exista, no necesita solo de corruptibles, sino también de corruptores. Esta afirmación, nuevamente evidente, acarrea un mensaje tácito: los problemas no son solo ellos, somos también nosotros» (Álvarez, 2013). 
Las causas de la corrupción, se clasifican en Causas formales, se concretan a falta de una clara delimitación entre lo público y lo privado, la existencia de un ordenamiento jurídico inadecuado a la realidad nacional, la inoperancia práctica de las instituciones públicas; causas culturales, causado por la existencia de una amplia tolerancia social hacia el goce de privilegios privados, permite que prevalezca una moralidad del lucro privado sobre la moralidad cívica, la existencia de una cultura de la ilegalidad generalizada o reducida a grupos sociales que saben que la ley no cuenta para ellos fomenta la corrupción y la tolerancia social hacia ella, la persistencia de formas de organización y de sistemas normativos tradicionales, enfrentados a un orden estatal moderno, suele provocar contradicciones que encuentran salida a través de la corrupción, para algunos casos latinoamericanos, ciertas manifestaciones corruptas podrían explicarse por la escasa vigencia de la idea de nación y la ausencia de una solidaridad amplia fundada en el bienestar común; causas materiales, se refieren a situaciones concretas que dan lugar a prácticas corruptas. Las situaciones concretas de las que se trata en este caso son las distintas brechas existentes entre el orden jurídico y el orden social vigente (Banfield, 1975).

Si bien desde otras ópticas la corrupción puede ser calificada como favorable o desfavorable, en términos económicos debería existir una preocupación por el crecimiento de la corrupción si ésta produce subdesarrollo y pobreza. Las consecuencias económicas de la corrupción son negativas. Este problema se refuerza en aquellas economías en las que los servidores públicos son relativamente mal pagados, pues es frecuente que una parte sustancial del ingreso provenga de exacciones que siendo legales no tienen como contrapartida servicios gubernamentales o abiertamente de prácticas indebidas, otro criterio establece que en sociedades donde las relaciones raciales, religiosas, de castas o tribales son importantes (por ejemplo, en África), los funcionarios públicos suelen estar obligados a repartir los ingresos de las oficinas públicas entre los miembros de su grupo de referencia (Seña y Francisco, 2002 ).

Sin embargo, los resultados de la investigación, evidencian que el 15\% de los encuestados que confesaron que se cumple de forma aceptable que el sistema de control realiza auditorías a los estados financieros y presupuestarios, son los que precisan que se cumple en mayor grado que la corrupción es producida por una alteración patológica de los valores colectivos de los funcionarios; el 23\% que manifesto que no se cumple que el sistema de control realiza auditorías de desempeño para determinar la eficacia, eficiencia, economía y calidad de la producción y entrega de bienes y servicios, son los que precisan que se cumple en mayor grado que la ineficiencia del estado se origina por los trámites y procedimientos burocráticos que reducen la agilidad y transparencia de la gestión pública; y el 22\% que aseveró que no se cumple que el sistema de control reali- 
za auditorías de cumplimiento para determinar si la entidad aplica la normativa, disposiciones internas y estipulaciones contractuales, son los que señalan que se cumple en mayor grado que es escasa la participación ciudadana en la vigilancia de los actos de los servidores públicos; del análisis documental se advierte que las sociedades de auditoría durante el periodo estudiado, realizaron 4 auditorías a la información financiera y a la información presupuestaria, la contraloría y el ORCI no realizaron ninguna auditoría de desempeño, la contraloría realizó 14 auditorías de cumplimiento y el ORCI 42. Por otro lado, el Gobierno Regional realizó 1,056 adquisiciones vía procesos de selección por S/1,036'209,790,06, y en cuanto a la implementación de recomendaciones de auditoría, el gobierno regional tuvo 2,160 recomendaciones en situación de «en proceso» y 963 recomendaciones en condición de "pendiente". De las entrevistas se tiene que el 25\% manifestó que la causa de la corrupción fue la impunidad, el 20\% explicó que se debió a la falta de valores, el 17\% expresó que fue el uso indebido de caudales, el 11\% sostuvo que se generó por la acción u omisión de los servidores y el 10\% cree que fue la falta de controles internos efectivos.

\section{CONCLUSIONES}

El control previo incide de manera insuficiente en la reducción de la corrupción política evidenciada en el Gobierno Regional de Áncash, lo que es concordante con el análisis estadístico que revela una $\mathrm{p}=0,003$, siendo este menor a 0,05 , por lo que el resultado es significativo, corroborándose que las variables estudiadas son dependientes en consecuencia, existen evidencias suficientes para decir que el control previo y la corrupción política se relacionan.

El control simultáneo impacta exiguamente en la disminución de la corrupción económica del Gobierno Regional de Áncash, lo que es coherente con el análisis estadístico que revela una $\mathrm{p}=0,002$, siendo este menor a 0,05 , por lo que el resultado es significativo, confirmándose que las variables estudiadas son dependientes en consecuencia, existen pruebas suficientes para decir que el control simultáneo y la corrupción económica se complementan.

El control posterior incide escasamente en la contracción de la corrupción social advertida en el Gobierno Regional de Áncash, lo que es compatible con el resultado estadístico que demuestra una $\mathrm{p}=0,001$, siendo este menor a 0,05 , por lo que es significativo, reafirmando que las variables estudiadas son dependientes, en consecuencia, existen pruebas suficientes para sostener que el control posterior y la corrupción social se complementan. 


\section{AGRADECIMIENTOS}

Al Sistema Nacional de Control integrados por la Contraloría General de la República, Órganos de Control Institucional y sociedades de auditoría, por permitirnos acceder a la información; y a los especialistas en control y sociólogos expertos en corrupción, por su colaboración desinteresada.

\section{REFERENCIAS BIBLIOGRÁFICAS}

Álvarez, Miguel. 2013. Corrupción social. Buenos Aires.

Banfield, Edward. 1975. Corruption as a feature of governmental organization. Journal of Law and Economics.

Cárdenas. 2010. «El sistema nacional de control y la confianza ciudadana». Tesis, Lima.

Congreso. 2002. «Ley Orgánica del Sistema Nacional de Control». Artículo 60, 2002: 225885.

Khoury, Fuad. 2014. Diario 16. Sistema de nacional de control y al corrupción. 〈http:/ /www. http://diario16.pe/noticia/47457-khoury-hay-un-problema- generalizado-corrupcion-delincuencia-ancash. [Consulta: 9-12-2015].

Seña, Malem y Francisco, Jorge. 2002. La corrupción: aspectos éticos, económicos, politicos y jurídicos. Barcelona: Gedisa.

North, Douglass. 1995. Instituciones, cambio institucional y desempeño económico. México: Fondo de cultura económica.

Robbins, S. y Coulter, M. 1994. Administration. 4th Edition. Mexico: Prentice Hall.

Sayed, Taleh y Bruce, David. 1998. Police corruption: towards a definition of work. London.

Recepción: 09/03/2018

Aceptación: 17/05/2018

\section{Correspondencia}

Javier Pedro Hidalgo Mejía

javierhidalgome@hotmail.com 